Is abella Benda-Weber

\title{
Die Reliefamphora von Mykonos: Ein Beitrag zur Trachtenkunde des 7. Jahrhunderts v. Chr.*
}

Die frühgriechische Zeit ist für den Trachtenforscher eine besonders interessante, spannt sie doch grob betrachtet den Bogen zwischen der geschneiderten minoisch-mykenischen Jacken-Rock-Tracht und den auf perfekten Faltenwurf ausgerichteten klassischen Gewändern - Peplos, Chiton und Himation. Nach den sog. Dunklen Jahrhunderten, welche die Forschung allerdings immer mehr zu erhellen vermag, beginnt das aussagekräftige Material in spätgeometrischer Zeit ${ }^{1}$ und wird im Wesentlichen erst im 7. Jahrhundert greifbar. Gerade in der orientalisierenden Epoche werden vielfältige Einflüsse - etwa aus der syrischen und späthethitischen Kunst - mit dem durchaus nicht in Vergessenheit geratenen spätmykenischen Erbe kombiniert ${ }^{2}$, wodurch sich neue, noch uneinheitliche Gewandformen im griechischen Kulturraum ausbilden. Aus der griechischen Frühzeit kennen wir keine Frauendarstellung, »die eindeutig mit dem drapierten und genestelten Peplos bekleidet ist $\aleph^{3}$. Das Bildmaterial weist das frühgriechische Gewand entweder als zusammengenähte Stoffröhre oder als ein aus einzelnen Teilen gefertigtes, also genähtes Kleid aus ${ }^{4}$.

Unter dem reichen Material von Gewanddarstellungen im 7. Jahrhundert v. Chr. verdient die Gattung der tenisch-böotischen Reliefkeramik besondere Aufmerksamkeit, da sie viele Hinweise auf die Kleidung jener Zeit liefert. Da die ältesten figürlichen Reliefamphoren auf Tenos gefunden wurden und sie in ihrer Gesamtkomposition durch und durch kykladisch sind, nimmt die Forschung als deren Schöpfer teniotische Wanderkünstler an ${ }^{5}$. »In der reichen Stempelung der Gewänder« macht sich »eine neue Prachtliebe bemerk-

* Zusätzlich zu den vom Österreichischen Archäologischen Institut vorgegebenen Abkürzungen $<$ http://www.oeai.at/publik/autoren. html> werden hier folgende verwendet:

Bieber 1928

Bieber 1934

M. Bieber, Griechische Kleidung (Berlin 1928).

Caskey 1976

M. Bieber, Entwicklungsgeschichte der griechischen Tracht (Berlin 1934).

Ervin 1963

Kontoleon 1970

M. E. Caskey, Notes on Relief Pithoi of the Tenian-Boiotian Group, AJA 80, 1976, 19-41.

M. Ervin, A Relief Pithos from Mykonos, ADelt 18A, 1963, 37-75.

Marinatos 1976

N. M. Kontoleon, Die frühgriechische Reliefkunst, ArchEph 1969 (1970) 215-236.

S. Marinatos, Kleidung, Haar- und Barttracht, ArchHom 1, Kap. A/B (Göttingen 1967).

Pekridou-Gorecki 1989 A. Pekridou-Gorecki, Mode im antiken Griechenland: Textile Fertigung und Kleidung (München 1989).

Schäfer 1957 J. Schäfer, Studien zu den griechischen Reliefpithoi des 8.-6. Jahrhunderts v. Chr. aus Kreta, Rhodos, Tenos und Boiotien (Kallmünz 1957).

Schefold $1964 \quad$ K. Schefold, Frühgriechische Sagenbilder (München 1964).

${ }^{1}$ Einige wenige Funde mit detaillierteren Gewanddarstellungen stammen aus früherer Zeit, wie die Göttin auf einem protogeometrischen Pithos aus Fortetsa (Archäologisches Museum Heraklion Inv. 1440; J. N. Coldstream, Geometric Greece [London 1977] 69 Abb. 21 b).

${ }^{2}$ Marinatos1967, A 15-32. 44-46. $51 \mathrm{f}$.

${ }^{3}$ Pekridou-Gorecki 1989, 90.

${ }^{4}$ Die korrekte Bezeichnung dafür kennen wir nicht. In der Literatur werden solche Gewänder wahlweise mit den Termini >Peplos` oder 〉Chiton〈 versehen, oder mit >daedalic tunic〈 umschrieben. »Chiton« heißt bei Homer nur das Gewand der Männer (Bieber 1934, 23), »sleeved peplos« (so Ervin 1963, 46) ist eine in sich widersprüchliche Bezeichnung und kann für ein geschnittenes und genähtes Ärmelgewand, z. T. auch mit Schleppe, nicht verwendet werden (Marinatos 1967, A 44). In diesem Sinne spricht auch A. Filges, Schlauchkleid - Peronatris - Stola: Die Genese einer Frauentracht, AA 2002/1, 259 für die spätere Zeit von figürlichen Darstellungen diverser Kunstgattungen, »deren Trachtenstücke bei näherer Analyse längst nicht so gut bekannt sind, wie es die Beschreibungen dieser Bildwerke häufig implizieren«.

${ }^{5}$ Schäfer 1957, 88. - Of all the classes of relief pithoi, those from the Cyclades were, on present evidence, the first with figured scenes.« (Caskey 1976, 30). 

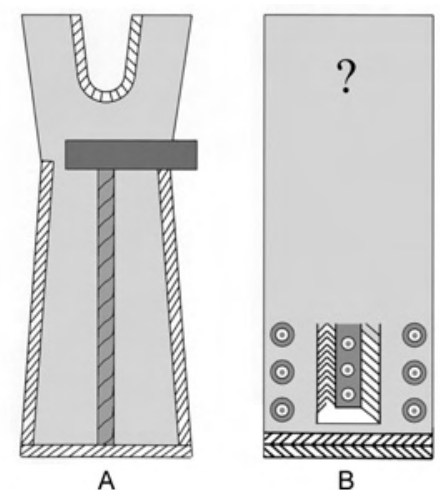

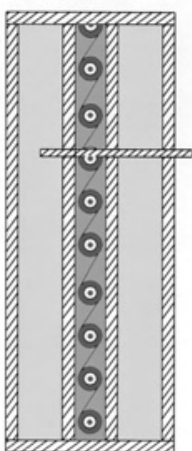

C
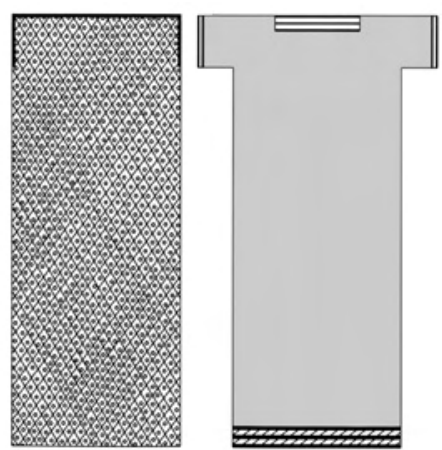

D

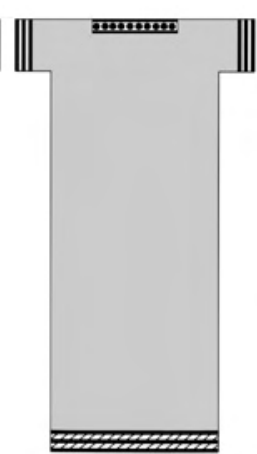

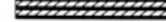

1 Schnittschemata von Frauengewändern tenisch-böotischer Reliefamphoren. A: Tanz-Amphora aus Tenos (Arch. Mus. Tinos); B: Fragment aus Tenos (Arch. Mus. Tinos); C: Fragment in Paris, Bibliothèque Nationale Inv. $64 \mathrm{C} 23563$ und sog. Ariane vom Halsfries der Tanz-Amphora aus Tenos (Arch. Mus. Tinos); D: Reliefamphora aus Theben (Athen, NM 5898)

bar«. ${ }^{6}$ Diese Technik ist für die Wiedergabe von Einzelheiten des Gewandes besonders geeignet, da sie eine Detailgestaltung erlaubt, die in der gleichzeitigen Vasenmalerei noch nicht erreicht wird.

Bei dem Fragment der sog. Tanz-Amphora aus Tenos ${ }^{7}$, die in das erste Viertel des 7. Jahrhunderts v. Chr. datiert wird, nützt der Künstler diese Möglichkeit auch in hohem Maße: »Niemals ist eine Figur die Wiederholung einer anderen. $\ll^{8}$

Vom Halsfries der Amphora ist nur ein Paar - wohl Ariadne und Theseus ${ }^{9}$ - erhalten. Der Rockteil des Frauengewandes ist an den Seiten und am Bodensaum von schraffierten Borten gesäumt (Abb. $1 \mathrm{C}$ ). Ab der eng gegürteten Taille verläuft ein senkrechter Mittelstreifen mit konzentrischen und durch Stege verbundenen Kreise, der von den Schraffurborten gerahmt wird. Das erheblich kürzere, ebenfalls eng gegürtete Gewand des Theseus ist ähnlich dargestellt: Eine schmale, schraffierte Mittelborte oder Ziernaht reicht bis zum Halssaum; in Brust- und Hüfthöhe sind einzelne Zierelemente (konzentrische Kreise) angebracht. Den Amphorenbauch schmücken drei Zonen mit Reigentänzern: In der ersten und dritten Zone tanzen Männer und Frauen abwechselnd gereiht nach links zur Musik eines Diauletes, in der zweiten Zone nur Frauen nach rechts. In der ersten und dritten Zone sind die Unterkörper der Tänzer und damit die Längen ihrer Gewänder nicht erhalten, die Männer sind aber von den Frauen durch die Gestaltung der Kleidung zu unterscheiden. Den geraden Halssaum, die schraffierten Saumborten und die bis zum Hals verlaufende Mittelborte weist auch das Gewand des Theseus auf, weswegen die Träger eines solchen Gewandes als Männer anzusprechen sind. Das Oberteil der Frauen ist hingegen bis auf einen etwa bis zur Brust reichenden, verzierten Bogen schmucklos. Da eine umgehängte Girlande wenig wahrscheinlich ist, kann es sich nur um einen gesäumten, bogenförmigen, weiten Halsausschnitt handeln, der allerdings in dieser Form einzigartig ist.

Auf einem gleichzeitigen Fragment aus $\operatorname{Tenos}^{10}$ ist der Unterkörper einer Frau erhalten, deren eng anliegendes Kleid am Bodensaum von einer breiteren Borte mit Fischgrätmuster abgeschlossen wird (Abb. 1 B). Die senkrechte Mittelborte mit konzentrischen Kreisen ist umlaufend von einer Borte gerahmt, die an der rechten Längsseite als Fischgrätmuster und an der linken als Schraffur gestaltet ist; an der Schmalseite ist nur die Außenlinie erhalten. Seitliche Außennähte sind nicht auszumachen, aber konzentrische Kreise füllen lose gereiht das Gewand.

In sehr ähnlicher Weise ist das Gewand der auf dem Stier reitenden Europa gestaltet (Abb. 1 C), die auf dem Fragment einer Reliefamphora in Paris erhalten ist ${ }^{11}$. M. Ervin schreibt dieses Stück dem Künstler der

\footnotetext{
${ }^{6}$ Schäfer $1957,75$.

7 Kontoleon 1970, 227 Taf. 48. 49. 50 b; Caskey 1976, 27.

8 Kontoleon 1970, 227.

9 Kontoleon 1970, 227.

10 Archäologisches Museum Tinos. Kontoleon 1970, 225 Taf. 42 b; Schäfer 1957, 71 Kat. T 9; Caskey $1976,27$.

${ }_{11}$ Paris, Bibliothèque Nationale Inv. 64C 23563. Schäfer 1957, 73 Kat. B 5; Schefold 1964, 29 Taf. 11 b.
} 


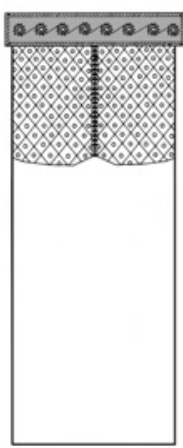

E

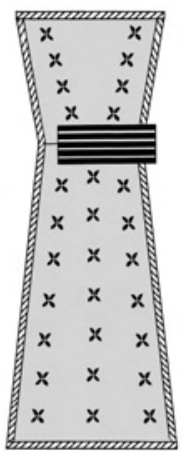

$\mathrm{F}$

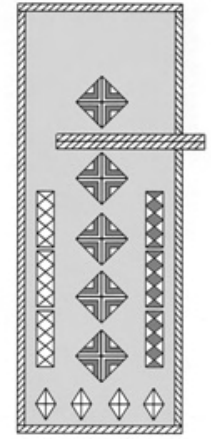

G
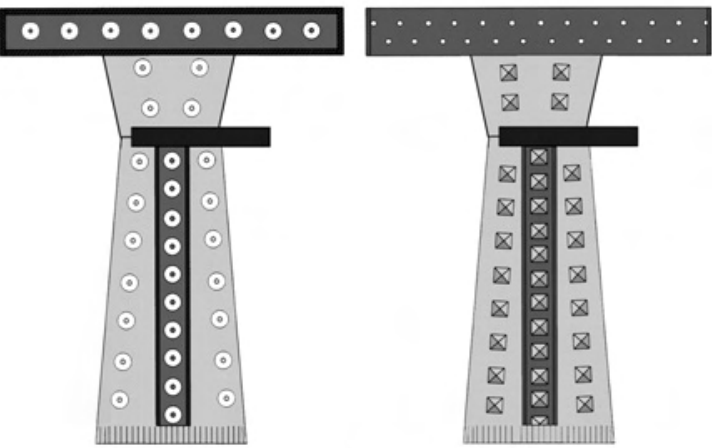

$\mathrm{H}$

2 Schnittschemata von Frauengewändern tenisch-böotischer Reliefamphoren. E: Fragment aus Tenos (Arch. Mus. Tinos), F: Fragment aus Tenos (Arch. Mus. Tinos), G: Amphora aus Tenos (Arch. Mus. Tinos), H: Amphora in Basel (Antikenmuseum BS 617)

Tanz-Amphora, dem sog. Ariadne-Meister, $\mathrm{zu}^{12}$. Auch hier sind die Seitennähte schraffiert wiedergegeben. Die senkrechte Mittelborte mit konzentrischen und durch Stege verbundenen Kreisen reicht über die Gürtung hinauf; Kopf und Schulterbereich fehlen. Die Gewänder der Frauen der Tanz-Amphora (Abb. 1 A), der Frau vom tenischen Fragment (Abb. 1 B) und der Europa (Abb. 1 C) unterscheiden sich nur unwesentlich.

Zwei Reliefamphoren, eine aus Theben sowie ein tenisches Fragment aus Xoburgo ${ }^{13}$, zeigen in ähnlicher Ikonographie jeweils eine weibliche Figur in frontaler Haltung, mit erhobenen Armen und flankiert von zwei kleineren Frauen, die als Potnia Theron mit zwei Adorantinnen gedeutet wird ${ }^{14}$. Das Gewand der Göttinnen fällt als ungegürtetes Rechteck zu den Füßen herab und ist bei beiden Darstellungen mit einem punktierten Rautenmuster verziert (Abb. 1 D). Das tenische Fragment ist hierbei detailreicher: Den oberen Saum bildet ein breiter Streifen, verziert mit elfblättrigen Blüten (Abb. 2 E). An allen vier Seiten ist diese Bordüre mit schmalen, schraffierten Bändern umrahmt und bildet mit ihrem Gegenstück der Rückseite die Ärmel. Das senkrecht in der Mitte verlaufende, fischgrätartige Band ist wohl als Ziernaht anzusehen, welche die zwei das Vorderteil bildenden Bahnen zusammenhält. Die Gewänder der tenischen Adorantinnen sind an allen Säumen mit dem Fischgrätband bzw. einer Ziernaht eingefasst. Diese ist auch am Rücken zu sehen - somit sind auch für den Rückenteil zwei Stoffbahnen, verziert mit kleinen Blüten, belegt. Die Begleiterinnen auf der Reliefamphora aus Theben tragen ein ungemustertes Kleid, das aber deutlich kurze Ärmel und doppelte Zierbänder (schraffiert oder punktiert) an den Säumen aufweist (Abb. 1 D).

Auch die weibliche Figur auf einem weiteren tenischen Fragment ${ }^{15}$ trägt ein rechteckiges, flächendeckend mit vierblättrigen Blüten geschmücktes Gewand (Abb. 2 F), das mit schraffierten Bändern umrahmt ist ${ }^{16}$. Hier ist es aber eng anliegend, geschnürt mit einem fünfzonigen Gürtel.

Die geflügelte kleinere Figur, die links neben der gebärenden Göttin auf dem Halsbild einer tenischen Geburts-Amphora ${ }^{17}$ zu erkennen ist, trägt ihr rechteckiges, von Schraffurborten gesäumtes Kleid nur schmal und keineswegs eng gegürtet (Abb. 2 G). Hier ist auch ein gerader Zuschnitt ohne Taillierung anzunehmen.

\footnotetext{
${ }_{12}$ Ervin 1963, 75.

${ }_{13}$ Reliefamphora aus Theben, Athen, NM 5898, und tenisches Fragment aus Xoburgo, Archäologisches Museum Tinos; Caskey 1976, 28 f. datiert sie in das 2. Viertel des 7. Jhs.

${ }^{14}$ Schäfer 1957, 71 Kat. T 10 und 73 Kat. B 1; Caskey 1976, 28. 29. 32 Taf. 4 Abb. 13.; Schefold 1964, 30 Taf. 12: Leto mit Eileithyien; Kontoleon 1970, 231 Taf. 56 f.: delische Artemis mit hyperboräischen Jungfrauen.

15 Schäfer 1957, 72 Kat. T 14. 82; Caskey 1976, 29; um 680/670 v. Chr.: LIMC I (1981) 80 Nr. 280 Taf. 85 s. v. Achilleus (A. Kossatz-Deissmann).

${ }^{16}$ Entweder kann man applizierte Zierborten oder aber Nähte voraussetzen, die bei dickeren Wollstoffen - ein solcher ist auch hier wahrscheinlich - beim Zusammennähen durchaus eine derartige Form annehmen können. Keinen Sinn macht die Interpretation als »fransenbesetzter Chiton« (so Schäfer 1957, 82).

${ }_{17}$ Archäologisches Museum Tinos. Caskey 1976, 27 datiert die Vase in das 1. Viertel des 7. Jhs.; Kontoleon 1970, 228 Taf. 52-55.
} 
Das Gewandmuster ist anders als bisher nicht flächendeckend, sondern mit einzelnen, in drei Längs- und einer Querreihe angeordneten Ornamenten (Sternen und Rautenbändern) dekoriert.

Dies ist auch bei den beiden gegen Minotauros kämpfenden Athenerinnen einer Baseler Reliefamphora ${ }^{18}$ der Fall, wobei aber die Dekorelemente der mittleren Reihe nicht einzeln, sondern auf einem Band gereiht sind, das in der Taille endet, während die beiden Reihen loser Elemente bis zu einem ebenso dekorierten Querstreifen am oberen Saum führen (Abb. 2 H). Er ist an die - gewiss tailliert geschnittenen - senkrechten Stoffbahnen wieder so angenäht, dass er den Halssaum und die halblangen Ärmel bildet. Der Querstreifen ist von schraffierten Bändern an allen Seiten eingefasst, seitliche Ziernähte am Kleid sind aber nicht zu sehen. Den Bodensaum zieren Fransen.

Reich mit Einzelornamenten übersät sind auch die prächtigen Gewänder der Trojanerinnen (Abb. 3 I) auf einer Bostoner Reliefamphora ${ }^{19}$. Die Gewänder der Dienerinnen weisen wiederum die schraffierten Seitennähte auf. Den Saum schließt eine Rautenbordüre ab. Die Oberteile sind leider nicht mehr erhalten, jedoch ist die ursprüngliche Gürtung noch zu erkennen. Die Ornamente sind meist kreisförmig, mit eingeschriebenen konzentrischen Kreisen, sechsblättrigen Blüten und anderen geometrischen und floralen Motiven. Einige davon erinnern an Goldapplikationen ${ }^{20}$, wie sie schon aus minoischer Zeit erhalten sind. Die Anordnung der Zierelemente ist immer noch dreireihig, aber aufgelöster und flächendeckend; die senkrechte Mittelborte fehlt. Die Frauengestalt, die den Zug anführt, ist durch ein Szepter, das längere Haar und vor allem durch die reichere Kleidung hervorgehoben. Sie trägt über dem Kleid, das als einziges eine Schleppe aufweist, einen reich verzierten Mantel, wieder mit diversen einzelnen, aber gereihten Ornamenten und doppelter Schraffurnaht.

Auf einer zweiten Reliefvase aus Boston ${ }^{21}$ ist auf dem Halsbild eine Szene mit einem Krieger und einer Frau, die eine in einem Tripod sitzenden Mann flankieren, fragmentarisch erhalten. Das Gewand der Frau ist im Oberteil mit punktiertem Rautenmuster, im Unterteil mit der senkrechten, von Punktreihen gerahmten Mittelborte verziert (Abb. 3 J). Der Schnitt zeigt eine Neuerung: Das Gewand ist vermutlich aus dünnerem Material vorzustellen, nicht tailliert geschnitten, aber eng gegürtet, weshalb der Kolpos seitlich über den Gürtel hängt.

Herausragend aus der Reihe der tenisch-böotischen Reliefvasen ist die Prunkvase aus Mykonos ${ }^{22}$, entstanden um 675 v. Chr., die in ihrer detailreichen Ausführung eine für diese frühe Zeit einzigartige Quelle für die Trachtenforschung darstellt (Abb. 4). Ihr Reliefschmuck erzählt vom nahenden Ende des Trojanischen Krieges, dem Schicksal der Frauen und Kinder von Troja - es handelt sich dabei um die älteste bekannte Darstellung der Ilioupersis ${ }^{23}$. Jede der Metopen der Bauchzone zeigt eine andere der Trojanerinnen, die mit verschiedenen Gestalten des Epos identifiziert werden ${ }^{24}$. Insgesamt sind 15 Frauengestalten soweit erhalten, dass Aussagen über ihre Kleidung möglich sind. Der Künstler nutzte die zahlreichen Personendarstellungen, um die prächtige Gewandung detail- und variantenreich auszuführen.

Alle Frauengewänder sind bodenlang, eng anliegend und tailliert, gegürtet, nach unten zu leicht ausgestellt, haben meist einen Fransensaum und eine Schleppe, Ärmel, die bis zum Ellbogen reichen, und einen hohen, horizontal verlaufenden Halssaum. Während die Gesamtsilhouette gleich bleibt, variieren Muster und

\footnotetext{
${ }_{18}^{18}$ Basel, Antikenmuseum BS 617. Schefold 1964, Taf. 25 a datiert die Amphora um 670/660, Caskey 1976, 29 f. in das 3. Viertel des 7. Jhs.

${ }^{19}$ Boston, Museum of Fine Arts 99.506. Abbildung der vollständigen Szene mit fünf Frauen als Umzeichnung bei A. de Ridder, Amphores Beotiennes à Reliefs, BCH 1898, 467 Abb. 10; Schäfer 1957, 82 Kat. B6; Schefold 1964, 42 Taf. 30 f. erkennt eine Opferprozession Hekabes und ihrer Dienerinnen mit feinen Gewändern für Athena; Caskey 1976, 29 Taf. 7 Abb. 23-26 datiert sie in das 3. Viertel des 7. Jhs.

${ }^{20}$ Die Gewänder der Bostoner >Hekabe〈 und ihrer Dienerinnen erinnern an eine von M. Bieber angeführte Homerstelle (Il. 6, 289-295), wonach »die kostbarsten Gewänder als Werke sidonischer Frauen gepriesen« werden, »so die ganz buntgemusterten, unter denen Hekabe das größte und durch seine Musterung allerschönste, das wie ein Stern leuchtet, als Gabe für Athena auswählt.« Vielleicht sind hiermit Goldapplikationen gemeint.

${ }^{21}$ Boston, Museum of Fine Arts 99.505. Von Caskey 1976, 29-30 Taf. 8 Abb. 33 in das 3. Viertel des 7. Jhs., von Schäfer 1957, $75-76$ um 625 v. Chr. datiert.

22 Archäologisches Museum Mykonos Inv. 2240.

${ }^{23}$ Ervin 1963, 57.

${ }^{24}$ Ervin 1963, 56-65.
} 

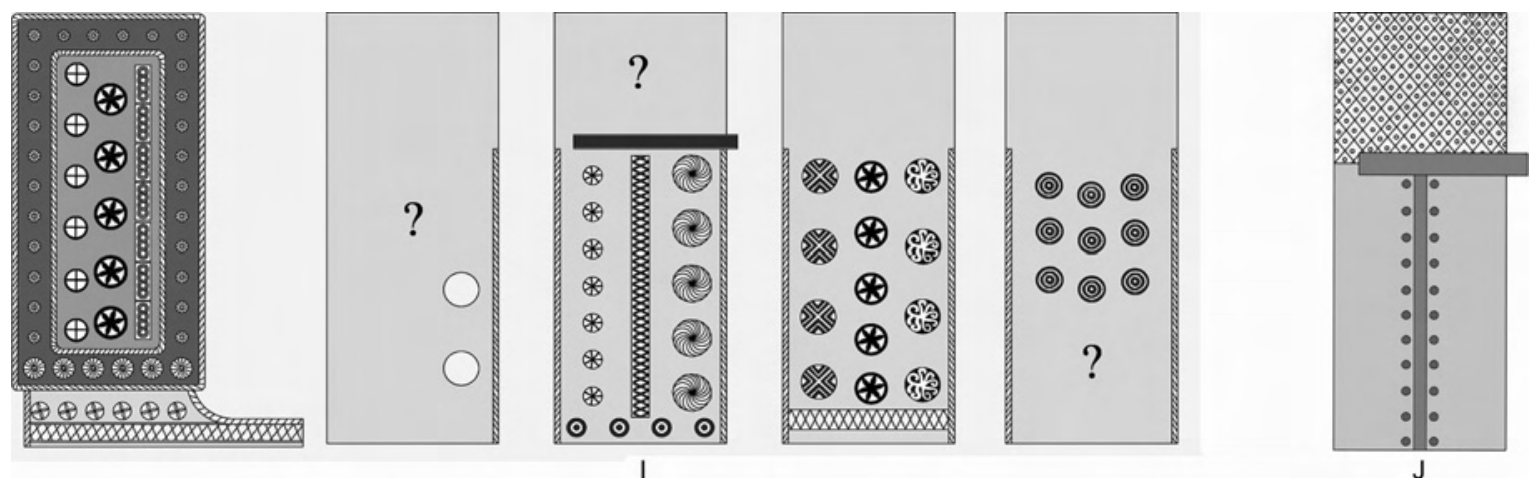

3 Schnittschemata von Frauengewändern tenisch-böotischer Reliefamphoren. I: Amphora in Boston (Museum of Fine Arts 99.506), J: Boston (Museum of Fine Arts 99.505)
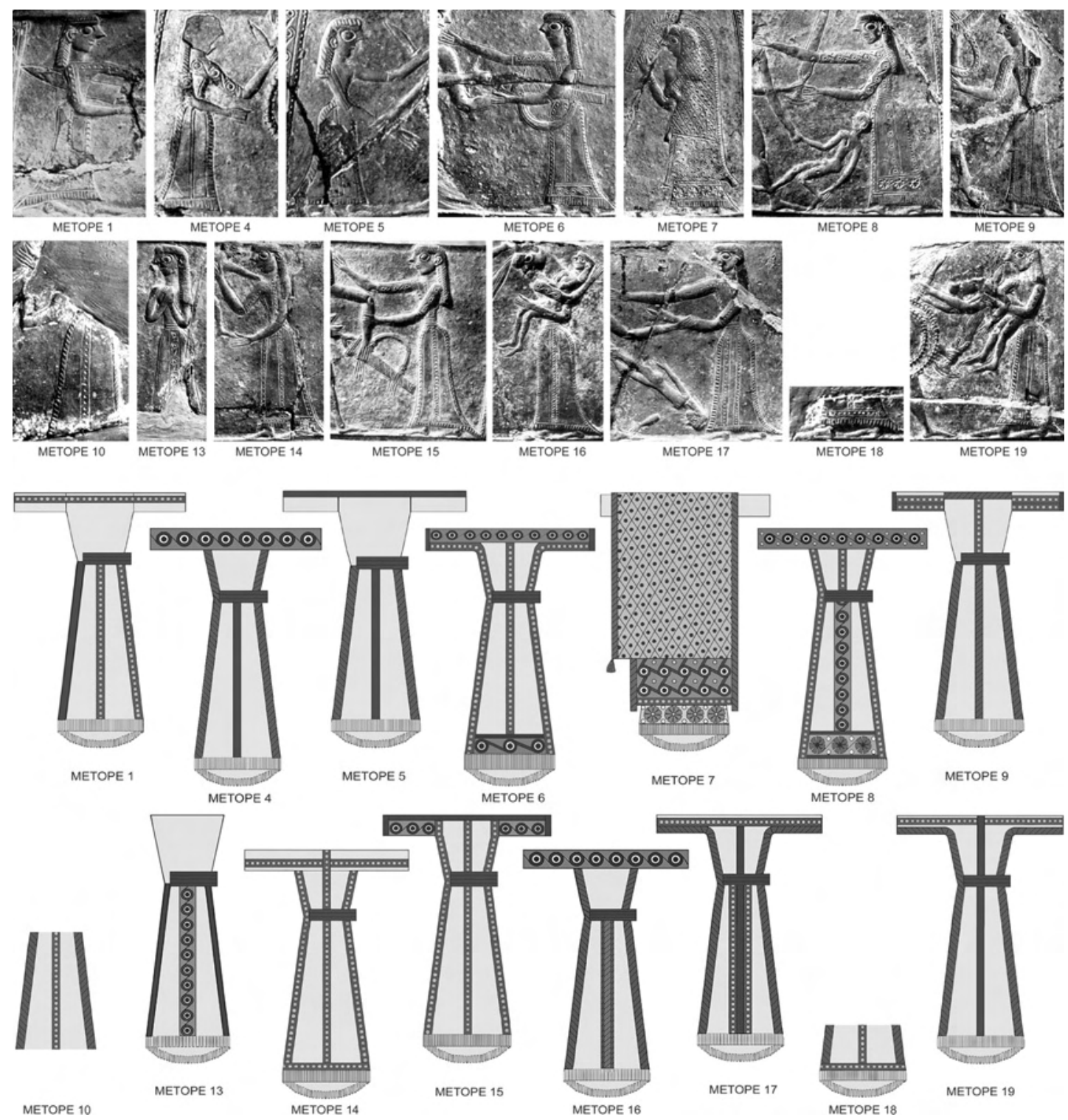

4 Synopse der Metopen 1-19 der Reliefamphora von Mykonos (Photos und Schnittschemata) 
Zuschnitt. Manche Gewänder sind prächtiger, manche etwas schlichter - offenbar gestaffelt nach sozialer Stellung oder Alter. Alle sind mit diversen Musterstempeln reich dekoriert und sehr ähnlich, doch kein Gewand ist dem anderen gleich. Die Frau von Metope 7 »stands out from all the others by the richness and detail of her clothing $\ll^{25}$. Als einzige trägt sie mehrere Kleidungstücke. Ihr Gewand ist »the most elaborate clothing of the pithos ... With each hand she holds an edge of her shawl, which is worn over her head and hangs down to below her waist. The front lower edge of the shawl has a tassel. Beneath the shawl she wears a himation, and beneath that a peplos. The border of the himation projects front and back, perhaps to represent tassels. At the back, the border of the shawl continues into that of the himation as if the two garments were one. ${ }^{26}$ Diese prächtig gekleidete Frau, die auch einen dreifachen Armreif trägt, kann wohl nur Helena meinen ${ }^{27}$.

Die Gewänder sind nicht aus einem Stück Stoff zu einer Röhre genäht, sondern setzen sich auf individuelle Weise aus mehreren Stoffteilen zusammen. Einige Kleider sind so geschnitten, dass ein horizontal verlaufendes, gemustertes Stoffrechteck von einem Ellbogen bis zum anderen reicht und so mit einem zweiten dahinter die Ärmel bildet (Metope 4. 6. 8. 16); bei Metope 14 ist das horizontal angesetzte Stoffstück unverziert, aber an der Oberkante von einer schmalen Borte begleitet. Die Ärmel des Kleides auf Metope 15 sind durch seitlich angesetzte Rechtecke aus dem gemusterten Stoff gebildet; das ist auch bei Metope 1, 5, 9 und 19 anzunehmen. Da sie aber aus demselben Stoff wie das Kleid gearbeitet sind, können die Ärmel auch angeschnitten sein, was zwar unwahrscheinlich, aber theoretisch möglich ist. Das Kleid von Metope 13 scheint ärmellos zu sein ${ }^{28}$ : Vertikale Stoffbahnen bilden das Kleid. Sind die Ärmel aus dem horizontalen, durchgehenden Stoffrechteck gebildet, sind die vertikalen Teile an dessen unterem Saum angenäht.

Der Dekor besteht aus Borten verschiedener Breite und Musterung. Es gibt schmälere Borten mit einer Punktreihe, mit Schraffur oder ohne Muster. Die breiten Stoffrechtecke, welche die Ärmel bilden und manchmal auch am unteren Saum angebracht sind (Metope 6. 7. 8) ${ }^{29}$, zieren konzentrische Kreise, die durch diagonale einfache oder doppelte Linien verbunden sind und so an eine laufende Spirale erinnern, oder mit achtzackigen Sternen oder siebenblättrigen Blüten gefüllte Kreise; manchmal sind Punkte in den Zwischenräumen zu sehen. Nur das Himation Helenas ist flächendeckend mit der laufenden Doppelspirale, das Schleiertuch mit Rautenmuster und Punkten geschmückt. Die Kombination der Musterborten ist sehr individuell, manche Kleider wirken bunter, während etwa das Kleid von Metope 14 mit einer Bortenart auskommt.

Die Borten verlaufen generell entlang der Säume und der Seitennähte sowie vertikal als Mittelstreifen. Dieser kann lediglich im Rockteil (Metope 1. 4. 5. 13. 16) oder durchgehend bis zur horizontalen Halssaumborte (Metope 9. 15. 17. 19), bis zum horizontalen Stoffstreifen (Metope 6 und 8) oder bis zur unverbrämten Stoffkante (Metope 14) verlaufen, oder ab der Mitte von einer anderen Borte weitergeführt werden (Metope 8). Bei den Kleidern auf den Metopen 1 und 8 entspricht der Mittelstreifen in Breite und Musterung dem, der bei manchen als Ärmelbordüren verwendet wird. Auf Metope 16 und 17 sind die Mittelborten dreifach nebeneinander genäht. Ebenso sind die Seitennähte entweder nur am Unterteil (Metope 1. 5. 9. 13) oder auch am Oberteil (Metope 4. 8. 14. 15. 16) von einer Borte verdeckt, oder aber diese ist an der Naht der Ärmelunterseite bis zum Ärmelsaum horizontal weitergeführt (Metope 6. 17. 19). Borten verlaufen auch von einem Ärmelende zum anderen und betonen so die Horizontale. Begleiten sie die Saumoberkante (Metope 1. 5. 15. 17. 19), verdecken sie die an der Oberseite liegende Ärmelnaht ${ }^{30}$ und bilden gleichzeitig den Halsausschnitt, der natürlich offen bleibt. Die Horizontalborten können aber auch etwas weiter nach unten versetzt sein, entlang der Vorderseite der Ärmel verlaufen (Metope 9. 14) und manchmal auch doppelt gelegt sein (Metope 9. 17). Auch an den Schmalseiten, also rund um die Ärmelöffnung, verläuft oft eine zarte Borte,

\footnotetext{
${ }^{25}$ Ervin 1963,61 .

${ }^{26}$ Ervin 1963, 48. - Zu den Gewändern s. auch Caskey 1976, 32: »The garments of the women, with decorated borders and panels, and tight fitting waists, are those worn in the Cyclades and elsewere at that time. Nikandre is dressed in this fashion, with the addition of an epiblema (...) The epiblema is usually missing on the pithoi, but this is more than balanced by the elaborate clothing of Helen on the Mykonos pithos (pl. 3, fig 16), equal to anything on the ladies of the >Melian` amphoras.«

${ }^{27}$ Ervin 1963, 61.

${ }^{28}$ Der Oberteil dieses Kleides ist überhaupt ohne Zierrat; es scheint sich auch um eine jüngere Frau zu handeln.

${ }^{29}$ Entweder ist er darauf appliziert, oder er schließt an die vertikalen Stoffstreifen an. Allerdings müsste dann der Fransensaum extra angenäht werden, weil er nicht vom vertikalen Stoffstreifen stammen kann, da dessen Kettfädenenden an den Schmalseiten sichtbar wären. Ob der Saum an der Rückseite ein Gegenstück hatte, ist unklar, technisch ist er jedenfalls nicht nötig.

${ }^{30}$ An der Ärmelunterseite ist bei Metope 1 und 5 keine Naht mehr nötig, es genügt ein Stoffbruch.
} 


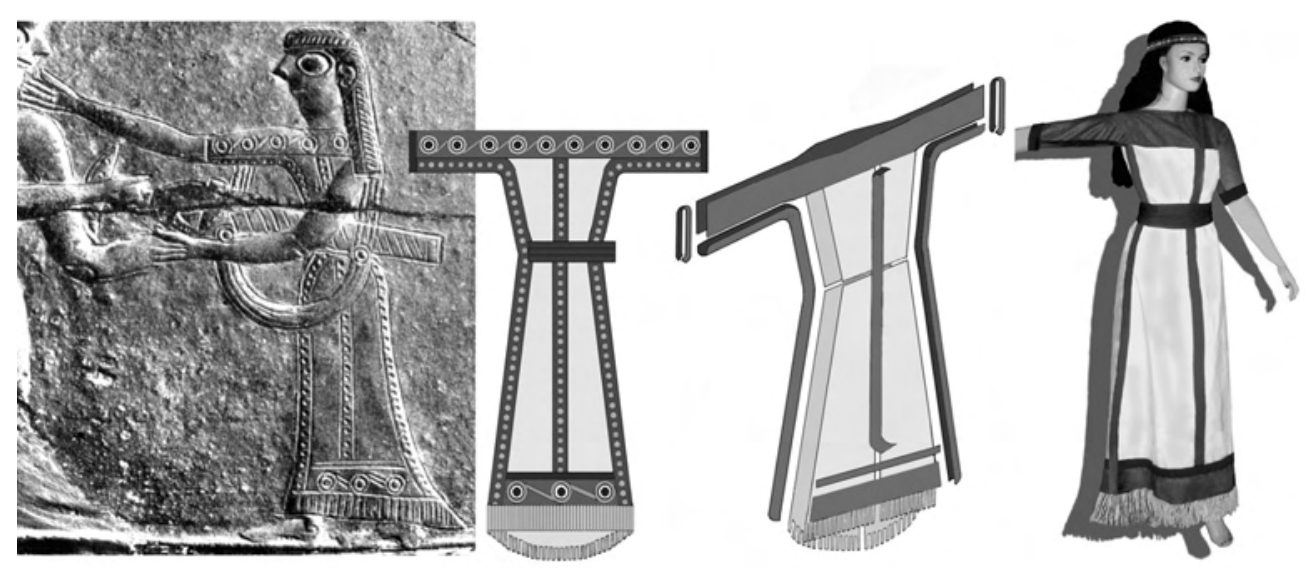

5 Metope 6 der Reliefamphora von Mykonos: Synopse von Photo, Schnittschema, Explosionszeichnung des Schnittes und Modellrekonstruktion

so erkennbar auf den Metopen 6, 9 und 15. Das Aufeinandertreffen unterschiedlicher Borten wird dabei recht kreativ gelöst: Bei Metope 14 und 19 wird die horizontale ausnahmsweise von der vertikalen Borte unterbrochen; bei Metope 9 sind die Borten an den Ärmeln und am Halssaum in Muster und Anordnung verschieden und verlaufen nicht durchgehend.

Wohl bei allen Kleidern wurde einen Fransensaum belassen ${ }^{31}$, der aus abgeschnittenen Kettfäden gebildet zu denken ist. Wie schon erwähnt, dient in manchen Fällen ein horizontales Stoffstück oberhalb der Saumkante zur Zier und zur Verstärkung des Stoffes. Eine schmale Borte kann parallel dazu noch an einer oder zwei Längsseiten verlaufen (Metope 6. 8); auch ohne die breite Bordüre bildet eine schmälere Borte manchmal die Trennlinie zwischen horizontalem Saum und Fransen. Die seitlichen Borten können dabei an die horizontal umlaufende Saumborte anstoßen (Metope 14. 18) oder umgekehrt (Metope 6. 8) ${ }^{32}$. Die Seitenborten von Metope 17 verlaufen bis zum Boden und unterbrechen so den Fransensaum. Bei Helenas Kleid (Metope 7) sind keine seitlichen Borten erkennbar, wahrscheinlich enden sie oberhalb der wohl umlaufenden, gemusterten Bordüre.

Eine Gürtung ist nicht immer zu erkennen, aber für alle Gewänder anzunehmen. Ein mehrfach (dreifach: Metope 8. 16. 17. 19; vierfach: Metope 13.15) gelegtes Band bildet eine breite Zone enger Schnürung an der Taille.

Ein Modellversuch (Abb. 5) soll eines dieser Gewänder der >Mykonos-Amphora - gewählt wurde Metope 6 - visualisieren, um zu prüfen, ob die oben erörterten Analysen auch in realiter funktionieren. Folgende Überlegungen gingen der Anfertigung voraus: Die Anordnung der Einzelteile und ihre deutlichen Begrenzungslinien und Stosskanten belegen, dass wir von geschneiderten und genähten Kleidern ausgehen müssen. Die schmalen Streifen sind oft umlaufend, müssen also nachträglich angebracht worden sein. Das schließt ein in einem Stück gewobenes Gewand aus. Borten folgen generell Nähten oder sind Nähte ${ }^{33}$. Sie dienen niemals nur der Zierde, sondern sind primär funktional, betonen die Struktur, dienen der Verstärkung und verdecken schiefe Nähte. Es handelt sich wohl um extra gefertigte Zierbänder ${ }^{34}$. Als Material für die Gewänder kommen sowohl Wolle als auch Leinen in Frage ${ }^{35}$. Die enge Passform legt einen Zuschnitt aus

\footnotetext{
${ }^{31}$ Im Einzelfall kann eine diagonal gestreifte Abschlussborte den Kleidersaum gebildet haben; der Unterschied zu den Fransen ist nicht immer erkennbar.

${ }^{32}$ Dort sind sie aber als Begleitung der breiten Bordüre zu verstehen oder bilden einen Bestandteil davon.

${ }^{33}$ In diesem Sinne auch A. Bönsch, Formengeschichte europäischer Kleidung (Wien 2001) 15.

${ }^{34}$ Möglich ist hier Fertigung in Brettchentechnik, aber auch an bestickte (importierte?) Bänder kann man denken. Von rezenten Trachtenhemden - etwa aus Thrakien, aus Kreta oder von den Sporaden - kennt man auch die Möglichkeit einer speziellen Ziernaht, mit der die Einzelteile zusammengenäht werden. Auch bei Homer (Od. 19, 242) ist ein mit einer Randborte (terma) versehener Chiton erwähnt (Bieber 1934, 23 f.).

${ }^{35}$ Für die Rekonstruktion wurde eine Mischform gewählt: Leinen für das Kleid, Wolle für die Borten und Bordüren. Die reiche Musterung musste aus Zeitgründen dabei vernachlässigt werden.
} 
mehreren Teilen ${ }^{36}$ nahe, neben den erwähnten Rechtecken für die Ärmel offenbar Trapeze oder Dreiecke, was der üblichen Vorgangsweise der vorindustriellen Trachtenfertigung entspricht. Der vom Webstuhl kommende rechteckige Stoff wird in geometrische Grundformen aufgeteilt: Durch Diagonalteilung können so zwei Trapeze oder Dreiecke ohne Verschnitt leicht hergestellt werden ${ }^{37}$. Der Schleppsaum ${ }^{38}$, den alle 15 Gewänder der Amphora aufweisen, entsteht durch einen längeren, vermutlich gerundeten hinteren Rockteil; gleichzeitig wird mehr Beinfreiheit bei bleibender schmaler Form erreicht. Die oft in der Körpermitte endenden mittleren und auch seitlichen Borten machen eine Taillennaht wahrscheinlich, die der breite Gürtel gut verdeckt. Wie erwähnt, folgen Borten im Allgemeinen Nähten, daher wird eine Mittelnaht wohl jeweils zwei Stoffteile, vorn und hinten, und wenn sie bis zum Halssaum verläuft, auch oben und unten, verbinden. Auch die an der Armunterseite verlaufenden Borten verbessern die Passform.

Es ist tatsächlich faszinierend, dass jedes Detail singulär ist, keines der Kleider ist mit einem anderen identisch, weder bezüglich Schnitt noch Musterung oder auch Anordnung der Borten. Dem Künstler war es offensichtlich ein Anliegen, auf diese Individualität hinzuweisen und alle Variationsmöglichkeiten auszuschöpfen, wobei er mit einfachen Mitteln wie den Musterstempeln eine lebendige Vielfalt auszudrücken vermag. Zweitens berücksichtigen diese Details auch nähtechnische Probleme. Der Meister hatte anscheinend reale Kleider als Vorbilder und verfügte zumindest über ein Grundverständnis in der Gewandfertigung. Offenbar war es ihm auch wichtig, die Informationen festzuhalten und zu vermitteln, dass zu seiner Zeit Prachtgewänder mit einiger Raffinesse gefertigt wurden ${ }^{39}$ und sich von der zusammengenähten Stoffröhre deutlich absetzen. Rückschlüsse auf tatsächlich existierende Gewänder sind wohl durchaus zulässig, bedürfen allerdings einer sorgfältigen Vorgangsweise ${ }^{40}$.

Kleidung hatte also schon im frühen 7. Jahrhundert als Ausdruck von wachsendem Luxus und regen Handelsbeziehungen im gesamten Mittelmeerraum einen hohen Stellenwert. Bei aller Vorliebe für prächtigen Zierrat, den die Meister der tenisch-böotischen Reliefvasen mit besonderer Sorgfalt wiederzugeben versuchten, bleiben aber die Grundstruktur des Gewandes - und damit eine gewisse Schlichtheit - sowie die individuelle Gestaltung immer erhalten, womit zwei wesentliche Charakteristika griechischer Kunst auch im Bereich der Kleidung und zu dieser Zeit schon vorhanden sind.

Dr. Isabella Benda-Weber

Österreichisches Archäologisches Institut, Franz-Klein-Gasse 1, A-1190 Wien

E-Mail: isabella.benda-weber@oeai.at

Abbildungsnachweis: Abb. 4. 5: Photos nach Ervin 1963, Taf. 20-26; alle Zeichnungen und Rekonstruktionen: Verf.

\footnotetext{
${ }^{36}$ »Als größte Webbreite können wir die annehmen, die eine Weberin bei sitzender Arbeitsweise mit ausgestreckten Händen erfassen kann.« (Bieber 1928, 8). Mehrere schmälere Stoffbahnen sind leichter herzustellen und zu bearbeiten, besonders wenn komplizierte Muster eingewoben (oder gestickt?) werden. »Das einzig erhaltene Stück Leinen aus klassischer Zeit weist eine Webbreite von ca. 0,50 m auf und entspricht damit einer Breite, die um 1600 in England und heute noch in Griechenland üblich ist.« (Marinatos 1967, A 6). Solch schmale Bahnen müssen mehrfach zusammengenäht werden.

${ }^{37}$ Bei mittelalterlichen und neuzeitlichen Volkstrachten wurden diese Dreiecke (Gere) seitlich an die Rechtecke angenäht, um die nötige Weite zu erzielen. Allein im griechischen Raum gibt es dafür unzählige Varianten; s. dazu die Synopse der Schnittmuster

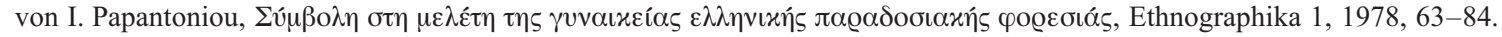

${ }^{38}$ Hom. Il. 5, 442; 7, 297; 22, 105 erwähnt solche Schleppkleider, allerdings nur bei Trojanerinnen, vielleicht auch explizit für Helena (Hom. Od. 4, 305). Es stellt sich nun die Frage, ob der >Mykonos-Meister von Troja Bezug nimmt, zumal auf anderen Reliefvasen die Schleppe nur selten vorkommt, oder ob er Gewänder aus seinem unmittelbaren Umfeld, also speziell kykladische Kleider, meint.

${ }^{39}$ Ein ebenso großes Streben nach individueller Gestaltung ist wohl auch für die tatsächlich geschneiderten Kleider anzunehmen. Alles in allem gewinnt man den Eindruck, als ob die Frauen das zur Verfügung stehende Stoff- und Bortenmaterial je nach Geschmack verwendeten, etwa so, dass die Borte, die von einer Trägerin als Mittelstreifen verwendet wird, der anderen als Ärmelabschluss besser gefällt. Vermutlich kommen wir der Realität mit solchen Vorstellungen recht nahe, entsprechen sie doch dem Prozess bei der Trachtenherstellung unserer Zeit, wobei sowohl lokale einheitliche Vorgaben der Tradition als auch der individuelle Gestaltungswille berücksichtigt werden.

${ }^{40}$ Im Vergleich zu der in vielerlei Hinsicht schematischen und der Stilstufe entsprechend von der Realität abweichenden Wiedergabe - etwa bezüglich Proportionen und Perspektive - ist die der Kleidung von einer erstaunlichen Präzision.
} 